\title{
Lectura y Gramática: la reflexión desde el verbo'
}

\author{
Reading and Grammar: Reflection from the Verb
}

\author{
MICAELA LORENZOTTI \\ LUISINA PIOVANO \\ CINTIA CARRIÓ \\ Universidad Nacional del Litoral \\ Argentina \\ lorenzottimicaela@gmail.com \\ luipiovano@hotmail.com \\ cincarrio@gmail.com
}

(Recibido: 09-O7-20I7; aceptado: II-O8-2OI7)

Resumen. En este trabajo se presenta un recorrido sobre los beneficios de la inclusión de la categoría gramatical "verbo" en el "aula de lengua" con la intención de instalar el debate desde un lugar propositivo. Las inquietudes surgen a partir del rastreo y análisis de estudios anteriores que hacen foco en el lugar de la gramática tanto en propuestas curriculares oficiales como en propuestas de "aulas de lengua" concretas. Se parte de la idea de que si bien la inclusión del contenido gramatical verbo está presente en las propuestas docentes, muchas veces la reflexión se ve relegada por otras urgencias como ser la corrección textual. En este trabajo se sostiene que para avanzar sobre la comprensión textual el trabajo con la gramática a nivel oracional es un paso ineludible, si bien no suficiente, para garantizar la comprensión de los textos y contribuir con el aprendizaje de la lectura crítica.

Palabras clave: didáctica; alfabetización; enseñanza; curriculum; gramática.
Abstract. The goal of this paper is to analyze and highlight the benefits of the inclusion of 'the verb' as a grammatical category into the 'language classroom'. This is done in order to bring the issue into analytical debate. Reflections appear as the result of tracing and analyzing previous research which focuses on the role of grammar on both official curriculum proposals and concrete proposals for 'the language classroom'. Although reflection about the verb -as a grammatical category- appears within teaching proposals, it is sometimes relegated by other needs such as text correction. This paper argues that in order to achieve effective text comprehension and to create and promote critical reading techniques, reflection on sentence grammar plays an essential role.

Keywords: didactics; literacy; education; curriculum; grammar.

\footnotetext{
${ }^{\text {I }}$ Para citar este artículo: Lorenzotti, Micaela; Piovano, Luisina y Carrió, Cintia (2018). Lectura y Gramática: la reflexión desde el verbo. Alabe 17. [www.revistaalabe.com]

DOI: IO.I5645/Alabe20I8.I7.9
} 


\section{Introducción}

Si se piensa, como se asume aquí, que la construcción de una propuesta de enseñanza está guiada por el concepto de "buena enseñanza" en el sentido en que propone Fenstermacher (I986: I58), entonces, el adjetivo "buena" remite a las justificaciones epistemológicas de la construcción curricular. Así entonces, requiere del planteo de preguntas respecto de qué contenidos son deseables y justificables (didáctica y epistemológicamente) que el docente, autor del curriculum, seleccione y reformule en su propuesta. En este sentido, parece deseable que el "docente autor del curriculum" (Gerbaudo, 2006: 69) se cuestione "[...] si lo que (se) enseña es racionalmente justificable y, en última instancia, digno de que el estudiante lo conozca, lo crea o lo entienda." (Fenstermacher, I986: I58)

Este artículo pretende instalar la discusión respecto de la selección de contenidos presentes en la curricula escolar y su relación con los objetivos pedagógicos y didácticos propuestos para el alumnado. Específicamente centra la atención en la importancia de la incorporación del contenido gramatical "verbo" y la urgente necesidad de revisión de su modo de abordaje en las clases, a fin de beneficiar una comprensión genuina de dicho contenido y un aprendizaje significativo en el marco general de los problemas de escritura y comprensión de textos.

Para avanzar en el desarrollo de este problema, hemos estructurado el texto de la siguiente manera: en el apartado siguiente $(\$ 2)$ se detalla la metodología que permitió recabar los datos para llevar adelante la investigación; en el apartado 3 se presentan las categorías analíticas centrales para la comprensión de nuestro planteo, así como las relaciones que asumimos entre dichas categorías; en el apartado 4 se desarrolla la discusión, se ofrecen precisiones sobre la categoría gramatical "verbo", se justifica la centralidad del contenido seleccionado (\$4.I) en relación con problemas que atañen al dominio oracional y extra-oracional (\$4.I.I) y en el último punto del apartado 4 se esbozan alternativas concretas de actividades que apunten a la consolidación de "buenas prácticas" (Gerbaudo, 2OII: 2O) (\$4.I.2); en el apartado final (\$5) se presentan las conclusiones.

Cabe aclarar en este punto que con "buenas prácticas", nos referimos a las intervenciones que llevan adelante los docentes en sus aulas, intervenciones atravesadas por decisiones tanto teóricas como epistemológicas. En tal sentido, asumimos que para lograr lectores y productores críticos es indispensable aspirar a una comprensión genuina (Perkins, I992) de ciertos contenidos centrales del área de lengua. En suma, desde nuestro punto de vista, la formación de un lector crítico (tanto de textos como de la realidad) supone conducirlo a comprender, o al menos a cuestionarse por las razones que conducen a los productores de ciertos fragmentos de lenguaje a preferir unas construcciones textuales o discursivas sobre otras, $y$, a la vez, evaluar el impacto que las decisiones de esa elección implican en relación con las intenciones explícitas o implícitas de quien produce. Reconocemos que es el docente de lengua quien tiene que acercarle al alumnado las herramientas que le permitan volver visible el mecanismo de poder vehiculizado por el lenguaje. 


\section{Metodología}

Es necesario resaltar que a la disciplina Lengua y Literatura le conciernen dos objetos de enseñanza. En concordancia con lo que sostiene Bombini (2OOI) consideramos que es necesario pensar en una "didáctica de doble objeto" teniendo en cuenta que la especificidad del objeto lengua y la especificidad del objeto literatura proponen un tratamiento didáctico particular, lo que implica que debemos devolverle a cada disciplina su identidad. Si tenemos en cuenta entonces esta particularidad debemos aclarar que nuestra propuesta se concentra exclusivamente en problemas vinculados con la didáctica de la lengua. Por esta razón, se indaga en las "aulas de lengua". Por "aulas de lengua" entendemos:

[...] no (...) sólo el diseño didáctico de la clase sino el conjunto de decisiones previas que se ponen en juego en cada una de esas actuaciones a lo largo de un período lectivo: la selección de contenidos, de materiales, los corpus (...), los envíos (...), el diseño de evaluaciones, la configuración didáctica (Litwin) de las clases. Este concepto llama la atención sobre el carácter artesanal y complejo de cada propuesta didáctica dado que cada grupo particular de alumnos requiere una re-selección y una composición didáctica de los contenidos pautados por los Ministerios (nacionales tanto como provinciales) [...] (Gerbaudo 2OII: 20)

Para llevar adelante el estudio, se seleccionó un método cualitativo. Por un lado se trabajó a partir del rastreo y análisis de estudios anteriores que hacen foco en el lugar de la gramática en las propuestas curriculares oficiales de Argentina, tanto en la ya derogada Ley Federal de Educación No 24I95 del año I993, como en la Ley de Educación Nacional No 26206 del año 2006 (Otañi y Gaspar 2OOI, 2008; Bassano y Freidenberg 2004; entre otros).

Por otro lado, las reflexiones de este escrito surgen a partir de los datos reunidos y analizados en Lorenzotti (2OI6), investigación en la que se indagó en una muestra de quince "propuestas de aulas de lengua" recopiladas a partir del trabajo de campo en instituciones de nivel medio de la ciudad de Santa Fe (Argentina). Uno de los interrogantes que se buscó responder mediante el análisis de las propuestas de "aulas de lengua" fue ¿qué lugar le asignaban los docentes como "autores del curriculum” (Gerbaudo, 2006: I5I) al "contenido gramatical verbo" en sus propuestas de "aulas de lengua"? Se arribó a la siguiente conclusión: las propuestas que incluyen al contenido gramatical "verbo" lo hacen en relación con la función que éste desempeña en los textos o con su función sintáctica como clase de palabra. Si bien en muchas de las propuestas se procuraba un espacio para el tratamiento léxico y para la reflexión gramatical sobre el contenido "verbo", esta reflexión quedaba generalmente relegada por otras urgencias como ser la corrección textual. De aquí surgen las inquietudes que le dan forma a este escrito. 


\section{Contenidos y objetivos}

Todo docente inicia el año de trabajo con un plan de acción. Al diseñar la secuencia escolar, la selección de los contenidos constituye un momento clave. La elección de los contenidos disciplinares en relación con los objetivos buscados requiere de un trabajo de prospección respecto de qué es lo que el docente espera que el alumnado pueda lograr a lo largo de ese año (los objetivos). De igual manera, la secuencia epistemológica, la selección de la teoría que permite pensar el objeto, la comprensión del acto y de la acción de enseñanza del objeto, son cuestionamientos que atraviesan la escritura de la propuesta para el aula. ¿Cómo se articulan los contenidos disciplinares con los contenidos pedagógicos y didácticos?; ¿qué se enseña en lengua?; ¿para qué se enseña lo que se enseña en lengua? y ¿̇por qué se enseña lengua? son algunos de los interrogantes que subyacen a esas propuestas.

Aquí se entiende por "contenidos" a aquello que se enseña, aquello que se decide enseñar y se incluye en el "plan", que tiene un tiempo y un espacio destinado para su tratamiento en el año. Los contenidos son los conceptos, categorías, operaciones, deducciones, explicaciones, que efectivamente se enseñan, que no se dan por supuestos, que no se espera que el alumnado deduzca solo. Los contenidos son aquellos conocimientos para los que se requieren instrucción, teorización, en el sentido de que el alumnado no puede saberlos sin que un otro se lo enseñe. Son los conceptos específicos que se desarrollan en el aula y que se espera que el otro aprenda genuinamente para activarlos en el momento en que lo requiera. Pero no sólo eso, un procedimiento también puede constituirse en contenido en la medida en que reciba un tratamiento como tal. Así entonces, un docente puede proponerse enseñar un procedimiento y en tal caso dicho procedimiento será un contenido a ser enseñando a lo largo de su plan de trabajo.

Ahora bien, un contenido es lo que se enseña en el aula independientemente de lo que se aprenda. En este punto asumimos, siguiendo a Fenstermacher, que si bien existe una relación muy estrecha entre la enseñanza y el aprendizaje "no se trata del tipo de conexión que apoya la afirmación de que no puede haber enseñanza sin aprendizaje” (I986: I52-I53), i.e. la enseñanza no implica en todos los casos, y como consecuencia directa o indirecta, el aprendizaje de algún tipo. Si la relación entre enseñanza y aprendizaje fuera directa e indisoluble, los desequilibrios cognitivos serían muchos más sencillos de resolver, sin lugar a dudas. Enseñanza y aprendizaje son dos fenómenos/procesos independientes, aunque obviamente relacionados.

La selección de contenidos en el área de lengua resulta en sí misma conflictiva. En Carrió (2008: 183), se postula una "crisis identitaria" que atraviesa el área Lengua y Literatura y que está basada en la falta de claridad respecto de "lo que se pretende" al momento de definir los objetivos y contenidos de la materia. Como consecuencia se genera un desfasaje entre lo "esperable" y lo "operable". Lo "esperable" es que un docente piense estrategias didácticas óptimas que les permitan al alumnado alcanzar una mejor 
comprensión de los contenidos seleccionados para el área. Lo "operable”, en cambio, es la incorporación en el aula de conceptos tomados (en algunos casos, reformulados) de alguna teoría lingüística. Dicha incorporación no supera el nivel de la definición y el reconocimiento, todo lo cual no conduce al alcance de los logros esperados con la enseñanza. Así por ejemplo:

La enseñanza del contenido 'tipo textual' por ejemplo [...] está desfasada en relación con el objetivo que se persigue a través de esa enseñanza. La enseñanza (y aún el aprendizaje) de los ‘tipos textuales’ o, más aún, de ‘los recursos de cohesión lineal' no tienen como consecuencia directa el aprendizaje de la escritura. No obstante, es lo que se espera que los alumnos logren con la ‘incorporación’ de ese conocimiento (otra discusión merecen las instancias didácticas que se proponen para presentar y ‘enseñar’ estos contenidos) (Carrió, 2008: I86)

Ahora bien, en una propuesta articulada, los contenidos seleccionados por el docente para ser enseñados tienen que entrar en diálogo con los objetivos planteados para cada grupo, a tal punto que el aprendizaje significativo de dichos contenidos permita el alcance de los objetivos formulados, es decir, de la meta final que visualiza el docente durante la selección curricular y a la que espera que arribe el estudiante luego del recorrido por los contenidos del programa. La importancia que le imprimimos al diálogo entre objetivo y contenido no es menor, consideramos que es la estructura que asegurará el éxito de la propuesta. Sin embargo, al momento de diseñar el año de trabajo, el docente no sólo proyecta el plan que discursiviza los objetivos y enumera los contenidos que van a posibilitar lograr los objetivos, sino que además, dicho plan de acción también contempla el modo en que se van a enseñar. Entonces, las actividades áulicas son el tipo de trabajo que día a día va a tener que enfrentar el alumnado para, recuperando contenidos del área y de su enciclopedia, buscar alternativas que le permitan resolver los problemas que el docente le plantea. En el plan de acción del aula, los objetivos, los contenidos y las actividades necesitan ser visiblemente coherentes y estar claramente cohesionados. De acuerdo a la propuesta, la reflexión en el aula girará en torno al reconocimiento de categorías, la reconstrucción de conceptos, la resolución de problemas, la evaluación de opiniones, etc. En términos de Perkins (I992:9I) apuntará a los diferentes “niveles de comprensión”.

$\mathrm{Al}$ referirnos a la importancia de la articulación objetivo-contenido-actividad hacemos hincapié en que la decisión que toma el docente en cuanto a la postura teóricoepistemológica que guiará la propuesta tiene que ser visible no sólo en los objetivos, lo que se discursiviza en una propuesta anual escrita, sino también en la forma de abordaje de los contenidos seleccionados. Lo que suele suceder con frecuencia es que objetivos constructivistas e incluso cognitivistas se asocian a actividades de reproducción y/o aplicación de recortes teóricos. En este sentido, reproducir definiciones no implica dominar las operaciones que esas definiciones recuperan. Por ejemplo, identificar los participantes de una situación comunicativa y definirlos teóricamente no tiene como co- 
rrelato directo mejorar la capacidad comunicativa, sino tener herramientas para realizar un análisis. Luego, si lo que se busca es trabajar la competencia comunicativa, probablemente serán más adecuados contenidos del tipo: reformulación de enunciados adecuados a situaciones específicas, lectura de implicaturas y sobreentendidos, detección de ironía y sarcasmos, reconocimiento de ambigüedades, entre muchos otros.

Que el docente se posicione teóricamente implica poder fundamentar la selección del contenido y del paradigma desde el cual concibe el objeto. La selección epistemológica de la teoría lingüística desde la cual se piensa el objeto trae consigo tanto una concepción acerca del lenguaje como del sujeto que aprende y la relación entre ambos. El posicionamiento lingüístico del docente va a determinar, entre otras cuestiones, el tipo de actividades que se proponga al alumnado.

Finalmente, un paso más de esta secuencia que el docente tiene que diagramar para su aula es la forma de evaluar los contenidos enseñados. Al referirnos a la evaluación, no lo hacemos en el sentido de la acreditación de saberes, de la cuantificación de lo aprendido, sino a una instancia más de aprendizaje en la que se recupera y se pone en juego lo ya trabajado en el aula. Las instancias de evaluación son constructivas en la medida en que se las piense como:

[...] evaluaciones que generen oportunidad de aprendizajes, promuevan la comprensión del propio proceso de conocimiento, posibiliten la articulación de saberes, se realicen con la intención de integrar y no excluir, permitan la enunciación de dudas, ignorancias e inseguridades, promuevan la participación de los involucrados, produzcan informaciones que estén al servicio de los protagonistas, se incluyan en los itinerarios curriculares, sean compatibles con el proceso de enseñanza y de aprendizaje, y permitan la toma de decisiones educativas respondiendo a los criterios de pertinencia y relevancia (Celman; Galarraga; Gerard; Grinóvero; Martínez; Olmedo y Rafaghelli, 2oI3: ı4)

La evaluación es una instancia de recuperación no sólo del contenido trabajado en el aula sino también de la forma en que fue abordado ese contenido en el devenir curricular. La evaluación es parte del proceso didáctico en tanto que implica para los estudiantes una toma de conciencia de los aprendizajes adquiridos y, para los docentes, una interpretación de las implicancias de la enseñanza de esos aprendizajes (Litwin, I998: I7). Sería deseable que las actividades evaluativas se diagramaran en función de tres premisas: (i) la evaluación tiene como objetivo la recuperación del contenido efectivamente enseñado durante el ciclo escolar (se evalúa lo enseñado y no lo supuesto); (ii) las actividades de evaluación propuestas tienen que estar en sintonía con la lógica de ejercitación de los contenidos enseñados (la instancia de evaluación no es el momento de la innovación, cambiar la operatividad de las consignas en la instancia evaluativa corre de foco el objetivo), y (iii) los estudiantes tienen que enfrentarse a situaciones que demanden un cierto esfuerzo cognitivo en las evaluaciones, lo que implica una forma de evaluación en la que 
los estudiantes no aprendan a leer un curriculum oculto, en la que no se concentren en tratar de descubrir las respuestas en función de lograr la promoción/acreditación sino para alcanzar una mejor comprensión.

\section{Precisiones sobre "verbo"}

Alisedo, Melgar y Chiocci (I994) definen a la didáctica de la lengua como una interdisciplina cuyas reflexiones teóricas integran aportes de otras disciplinas de referencia o de apoyo. Estas disciplinas conciernen tanto al contenido de la enseñanza, el contenido lingüístico -qué enseñar, qué aprender- como a los procesos de enseñanza, aprendizaje, adquisición y contextualización social que pueden aportar orientaciones acerca de cómo, cuándo, por qué y para qué enseñar.

Gerbaudo ubica el trabajo didáctico de la lengua (como así también al de la literatura) en una "zona de borde" que exhibe la conjunción de disciplinas necesarias para abordar problemas de la esfera educativa. Actúa así la transdisciplinariedad, es decir, "la confluencia de categorías y formas de resolución aportadas por distintos campos que se potencian para el análisis de ciertas cuestiones." (2OII: 25) Repensando esta noción de una didáctica de la lengua como transdisciplina a la hora de planear un "aula de lengua" se hace imprescindible buscar respuestas a algunos interrogantes, uno de ellos es ¿qué contenidos es "deseable" enseñar en el aula de lengua?

Asumimos que: (i) el objeto de conocimiento/estudio en el aula de lengua es el lenguaje y (ii) que los estudiantes son sujetos lingüísticamente creativos, y es en el aula donde la lengua se convierte en objeto de estudio y reflexión. En este sentido consideramos al lenguaje como una capacidad propia del sujeto del aprendizaje, capacidad de dominio no consiente (conocimiento implícito), que le posibilita la creatividad lingüística, i.e. construir un conjunto infinito de expresiones a partir de un conjunto finito de unidades y reglas de combinación, "[...] la llamada 'gramática mental' o 'competencia lingüística' que poseen los hablantes sobre su lengua nativa y que los hace capaces de comprender y producir un número infinito de enunciados.” (Defagó, 2OII: I89)

Si los conocimientos que tienen los estudiantes sobre su lengua son implícitos, cobra relevancia la pregunta respecto de por qué es necesario enseñar lengua. Bassano y Freidenberg (2004) argumentan que para formar un sujeto alfabetizado es necesario estudiar una lengua, entendiendo por estudiarla reflexionar acerca del conocimiento gramatical implícito del sujeto. La respuesta entonces podría sintetizarse en que es necesario enseñar lengua para aprovechar/potenciar esos conocimientos implícitos que el alumnado posee. Ahora bien, para arribar al objetivo, es fundamental hacer que esos conocimientos implícitos se hagan explícitos, es necesario ponerlos en evidencia, manipularlos, cuestionarlos, propiciar la enseñanza del lenguaje como un "proceso de explicitación” (Defagó, 20II: I89). Así entonces se vuelve relevante cuestionarse respecto de los contenidos que es "deseable" enseñar en el aula de lengua atendiendo al objetivo propuesto. 
Sostenemos, en consonancia con Carrió y Guglielmelli (2OII) que si tenemos en cuenta el objetivo de hacer explícitos los conocimientos implícitos que el estudiante tiene sobre su lengua para poder formar un sujeto alfabetizado, se torna necesario reconsiderar el espacio curricular lengua como un espacio en el que se propicie el conocimiento reflexivo sobre el lenguaje:

Consideramos que si se "entrena" a los sujetos en la "reflexión lingüística”, para a partir de allí, enseñarles a "mirar” el lenguaje como un objeto-instrumento en absoluto inocente y se presenta este modo de "representación" como un segundo mecanismo para desocultar los "no-dichos-lingüísticos" y las "implicaturas de los enunciados”, entonces se los está acercando a una lógica de razonamiento crítico, objetivo al que debe apuntar la escolaridad (Carrió y Guglielmelli, 2OII: 2IO)

La manipulación consciente de las estructuras discursivas (entiéndase por tales a las estructuras oracionales y también a las textuales) favorece la comprensión de los textos, el análisis crítico de los discursos, el desocultamiento de las estrategias de poder, el control de los actos de habla, entre muchos otros fenómenos atravesados por el lenguaje.

El docente como autor del currículum selecciona aquellos contenidos que considera "digno(s) de que el estudiante lo(s) conozca, lo(s) crea o lo(s) entienda" (Fenstermacher, I986: I5 $^{8}$ ) en función de los objetivos diagramados para la propuesta. Frente a esta elección que se realiza en relación con los contenidos, se puede hablar de una toma de posición de los docentes en tanto que son ellos quienes deciden qué se va a enseñar, qué visión de la realidad se adoptará, cómo se va a enseñar, entre otras cosas.

En este sentido, si se quiere atender en el aula de lengua a un objetivo que explote/explore la reflexión metalingüística de los estudiantes resulta entonces pertinente seleccionar contenidos relacionados con los conocimientos gramaticales. Entendemos que el conocimiento del léxico "puede y debe ser el punto de partida para la comprensión y producción de infinitas estructuras de una lengua natural” (Ciminari y Kocak, 2002: 7) Desde el punto de vista lingüístico y pedagógico resulta relevante incluir al contenido gramatical "verbo" en las propuestas curriculares del área lengua como punto de partida en la reflexión gramatical.

\section{I. "Verbo": justificación de su inclusión}

Si el objetivo propuesto para el aula de lengua es el de propiciar la reflexión metalingüística de los estudiantes para hacer explícitos sus conocimientos sobre el lenguaje, ¿por qué es pertinente seleccionar el contenido gramatical "verbo"? El verbo es una categoría léxica que se proyecta en la sintaxis en una construcción predicativa, en español además de presentar las marcas de tiempo, modo y aspecto, presenta los rasgos flexionales de persona y número, los que permiten pensar la operación sintáctica de la concordancia con el sujeto. En español la categoría gramatical verbo es la que permite relacionar 
el resto de la información en una oración simple (una estructura completa); es una de las categorías, si bien no la única, que hace posible la predicación: decir algo acerca de las entidades y de las relaciones que establecen dichas entidades entre sí o con los espacios.

Como expresión predicativa, el verbo pleno necesita descargar su estructura argumental para que se logre una oración bien formada. Determina cuántos y cuáles participantes son necesarios en la construcción, y describe la relación que se instaura entre ellos:

El predicado es como el guion de una obra: determina cuántos participantes se requieren y asigna a cada uno un determinado papel. De la estructura argumental depende qué elementos son obligatorios para formar una oración. Como se advierte, la relación entre el léxico y la gramática es muy íntima: en última instancia, la cláusula es una proyección del predicado semántico; por lo general, entonces, del verbo (Di Tullio, I998: 62)

Una de las aspiraciones de la escolaridad es trabajar en la mejora de la producción de textos, tanto orales como escritos. En este recorrido de búsqueda de perfeccionamiento, la categoría gramatical "verbo" resulta ser un contenido ineludible al momento de trabajar en el aula. Este posicionamiento no implica reducir el planteo didáctico a una dimensión sintactista, por el contrario, concebimos que la riqueza de su enseñanza radica en que a partir de la categoría verbo el alumnado puede problematizar la construcción y el ordenamiento de la oración simple. Luego y como consecuencia, se optimizará la construcción textual dado que la reflexión sobre esta categoría léxica incluye tanto el plano sintáctico en el que se proyecta, como la semántica involucrada en su carga conceptual y las flexiones morfológicas que hospeda.

\section{I.I. Intra y extra oracional}

Si consideramos entonces que el "verbo" es el nudo a partir del cual se establece la red de relaciones que conforman el mensaje, en lugar de establecer correspondencias entre estructuras y taxonomías se podrían propiciar los espacios para que el alumnado pudiera pensar, a partir de sus conocimientos intuitivos, sobre las propiedades del verbo.

Defagó y Guglielmelli (2005) explican que la información semántica del verbo es un aspecto que se puede manipular conscientemente y sirve como punto de partida para el análisis. Por lo tanto, desde el conocimiento semántico se podría repensar, explicar y determinar cómo se organiza la oración en torno al verbo, es decir, qué sintagmas tienen que ensamblarse según qué tipo de verbo se trate, qué papel semántico debe desempeñar cada uno de esos sintagmas en la oración y en qué estructuras sintácticas deben ordenarse.

A partir de la enseñanza del contenido "verbo" en el aula de lengua se puede reflexionar sobre la construcción textual porque en la construcción de los textos ciertas opciones gramaticales iniciales, como la selección de tiempos verbales, determinan la estructura del texto: "los tiempos verbales son fenómenos gramaticales y desde allí llegan al texto, y no viceversa.” (Demonte, 2005: 183) 
El conocimiento sobre la categoría gramatical "verbo" facilita la reflexión sobre una serie de fenómenos lingüísticos más complejos que la mera categorización. Así, el contar con información ordenada sobre la categoría "verbo" permite: I) reflexionar sobre los requerimientos de su formación y combinación; 2) construir generalizaciones sobre procesos derivativos a partir de la morfología; 3 ) explicitar las relaciones de concordancia entre el sujeto y el verbo atendiendo a la relación entre esta categoría y la de "nombre"; 4) atender a la exigencia de la estructura de los verbos transitivos que demandan necesariamente de un complemento compatible semántica y aspectualmente.

Así por ejemplo, “el saber sobre las correlaciones verbales permite evaluar su uso adecuado en los textos, y por lo tanto, la coherencia de éstos” (Otañi y Gaspar, 2oor: IO3). Más aún, desde una perspectiva sintáctica resulta importante distinguir entre elementos obligatorios y opcionales y "saber cuáles son las relaciones semánticas que se establecen entre las categorías que constituyen una oración.” (Llambí de Adra, 2005: 4) Incluso, si se considerara por ejemplo el caso de los papeles temáticos, contenido de gran importancia para la comprensión de los textos, se contaría entonces con una puerta de entrada consistente para el análisis semántico de los protagonistas textuales.

[...] las cuestiones relativas a las clases de palabras no son pertinentes sólo para la gramática sino que inciden directamente en el uso de la lengua y su desconocimiento está en la base de muchos errores comunes de los estudiantes, estas nociones (...) son de suma utilidad para mejorar la producción de textos en los diferentes ciclos de escolarización (Giammatteo y Albano, 2009: 69)

\section{I.2. Actividades}

La reflexión sobre la gramática a nivel oracional no es suficiente para garantizar la comprensión de los textos y contribuir con el aprendizaje de la lectura pero es un paso ineludible. Este tipo de reflexiones brinda al alumnado herramientas que, llegado el caso, les permiten manipular las formas lingüísticas para así reconstruir sentidos. Consideramos que la gramática no tiene que pensarse como un conocimiento subordinado al texto sino que es necesario reconocer la importancia del dominio gramatical al momento de producir y decodificar enunciados y textos.

Durante un tiempo prolongado, en Argentina se pensó (aun tácitamente) para este tipo de análisis, una enseñanza basada en la gramática estructural. Se habilitaron así malas interpretaciones de dicha mirada gramatical, instalando en el aula contenidos y categorías sin adecuaciones didácticas acordes a la situación. Se generó una especie de traslación acrítica de contenidos y análisis para los cuales no se tuvieron en cuenta las variantes que atraviesan la realidad educativa. Se instaló en el aula una gramática diseñada para fines no-escolares, y se le demandaron logros propiamente escolares.

En este sentido, se redujo la reflexión gramatical a la localización de categorías (sujeto y predicado, principalmente) y el etiquetado de segmentos oracionales (fundamentalmente objetos directo e indirecto y circunstanciales con clasificaciones semánti- 
cas). Este tipo de transposición didáctica, más cercana a la aplicación de teorías a fragmentos de lenguaje que a transposiciones basadas en la adecuación a las necesidades particulares del alumnado, cosechó no pocas críticas y enemigos en el ámbito educativo. Desde la transitada pregunta respecto de "¿y para qué nos sirve etiquetar constituyentes?" hasta "esto no me permite pensar nada", todo contribuyó a desencadenar el destierro de la gramática oracional de los programas de estudio de nivel primario y secundario.

Consideramos que estas críticas dirigidas a la gramática oracional (estructuralista, para estos casos a los que nos referimos) confunden el eje de la discusión y centran las críticas en una propuesta gramatical particular y no en una propuesta didáctica de enseñanza de la lengua. Concretamente, consideramos que las falencias radicaron/radican en la manera en que se piensa cómo volver accesibles los conocimientos al alumnado basándose en un conocimiento solido del objeto disciplinar.

Los hechos narrados se combinaron con la tendencia a presentar el análisis de "oraciones de juguete", es decir, oraciones especialmente pensadas para la localización de las categorías, pero, muchas veces alejadas de verdaderos fragmentos de habla y de discurso (esto así, ya sea por la selección léxica contenida como por el orden preferentemente no marcado en que se presentan los constituyentes, la tendencia a evitar oraciones marcadas por foco, la preferencia absoluta por el análisis de la variedad de lengua estándar, etc.). En palabras de Desinano (I997) se escolarizan contenidos y ejemplos, lo cual conduce al alumnado a restar significación a lo enseñado.

Desde nuestro punto de vista, si el macro objetivo del área de lengua radica, aunque no exclusivamente, en propiciar reflexiones a diferentes niveles gramaticales, entonces, la tarea requerirá no sólo de una adecuación de los contenidos a los sujetos de aprendizaje, sino además, una selección conforme con los micro-objetivos. Más aún, requerirá de una formación docente que permita alternativas en la elección. En tal sentido, los programas de formación docente demandan la incorporación de teorías que hagan posible pensar los problemas propios del lenguaje no sólo desde diferentes niveles de análisis sino también, y considerando un mismo nivel, desde múltiples perspectivas.

Son numerosos los trabajos que coinciden en describir la situación del ingresante de las carreras de profesorados de los distintos niveles como sujetos que han transitado largos períodos de escolaridad sin lograr un desarrollo aceptable de sus capacidades de producción y comprensión de textos (véase: Defagó, 2002; Defagó y Guglielmelli, 2005). Como plantea Castellà (I994), en el aula se necesita de una gramática pedagógica, ahora bien, dicha gramática no ha sido diseñada. Así entonces, el docente de lengua tendrá como desafío transformar un discurso lingüístico-científico en un discurso lingüístico-enseñable sin banalizar el objeto sobre el cual versan ambos tipos de discursos. En suma, se persiguen dos metas: I. volver enseñable el objeto, y 2. volver comprensible el discurso sobre el objeto.

Por ejemplo, es posible pensar en que a partir de las intuiciones que se tienen sobre algunas palabras y construcciones (en tanto que hablantes de una lengua) puede reconstruirse con poco esfuerzo la estructura argumental de los verbos. Para propiciar 
este razonamiento habrá que advertirles que atiendan a los verbos porque a partir de ellos se puede reconstruir con mayor facilidad la organización de la oración. Si se muestra el modo en que las oraciones describen situaciones y que en toda situación intervienen participantes (lo que teóricamente mencionamos como "argumentos del verbo"), entonces, a partir del verbo será necesario localizar los participantes de la situación que se describe (los actores de la situación supuesta por el verbo en cuestión). Si se trabaja con una oración que contiene el verbo "trasladar", será requisito indispensable para garantizar la buena comprensión del mensaje contenido la localización de los tres participantes implicados (los tres argumentos verbales). En términos simples entonces: quién traslada, qué se traslada, hacia dónde se traslada.

Si bien una actividad de este tipo puede parecer muy simple, (supongamos una oración sencilla del tipo: "El repartidor trasladó el paquete hasta el edificio"), hay casos en los que puede resultar más dificultoso ("Hasta el edificio equivocado trasladó aquel repartidor que recién habían contratado en la empresa de encomiendas que me recomendaste, el paquete que contenía todo el trabajo que realicé durante mi vida entera.”), sobre todo para aquellos que no cuentan con ningún tipo de estrategia para ordenar la (para ellos) desordenada sucesión de palabras que constituyen un texto. Así entonces, habrá que advertirles (“enseñarles a mirar”) que, algunas veces, los verbos tienden algunas "trampas" dado que no se bastan a sí mismos para expresar (significar) lo que realmente se quiere transmitir, entonces seleccionan otros verbos con la misma forma, o con otra, y al combinarse constituyen una única expresión (perífrasis verbales) que tiene más de un elemento y que no puede separarse para significar "eso" que se quiere expresar (supongamos: "alguien iba a trasladar algo a algún lado"). Más aún, otras veces, estos mismos verbos en lugar de seleccionar otro verbo, seleccionan construcciones no verbales (locuciones verbales). Entonces, en algunos casos (textos de algunas disciplinas) con mayor énfasis que en otros, habrá que atender también especialmente a las locuciones y construcciones metafóricas, tanto como a la modalización de esas construcciones.

Si se logra que el alumnado pueda acceder a la descripción de las situaciones que se van tejiendo en el texto, podrá haber más posibilidades de que acceda al sentido enunciado en el mismo y lo ponga en relación con el contexto de producción, de circulación y de recepción.

Para que sea posible establecer relaciones entre el nivel intra-textual y el nivel extra-textual, será necesario que el alumnado tenga a su alcance prácticas que le faciliten acceder a esa dimensión interna del texto porque, de lo contrario, si la concentración solamente está circunscrita al conocimiento contextual o a la macroestructura textual, entonces la lectura se basará sólo en especulaciones sobre el contenido, basado probablemente en la lectura de los paratextos.

Incluso en la formación de formadores resulta imperioso trabajar para contrarrestar la fuerza del aprendizaje ritual, la fuerza de la reproducción sobre la creación, de manera tal que los docentes basen sus decisiones tanto didácticas como epistemológicas en un conocimiento justificado en el conocimiento sobre el objeto y sobre el contexto 
áulico. De esta manera resulta posible instalar nuevas propuestas que tiendan a superar lo que, en principio, ya se sabe que no rinde los frutos esperados. Así pues, parece ser hora de reconsiderar los espacios y re-definir el espacio curricular, los objetivos y los contenidos para lograr una "buena enseñanza" en el aula de lengua que la destituya de un lugar servil e instrumental (cfr. Defagó (2002, 2OII); Demonte (2005); Llambí de Adra (2005); Otañi y Gaspar (2OOI)).

\section{Conclusión}

Para concluir entonces, afirmamos que el corrimiento de los contenidos gramaticales y con ellos la reflexión sobre el verbo de las curriculas escolares responde a malentendidos respecto de las maneras de pensar la transposición didáctica de la selección curricular. En las aulas de los niveles primario y secundario se reduce la reflexión gramatical a la aplicación de fórmulas de reconocimiento categorial o funcional, tal como si se tratara de un alumnado constituido por especialistas en lugar de sujetos ávidos por comprender e intervenir lingüísticamente la realidad.

A lo largo de este trabajo se argumentó a favor de las potencialidades de la reflexión gramatical minuciosa en torno a la categoría verbal, atendiendo a su carácter nuclear y, por tanto organizador de los mensajes. Se propuso el análisis de la estructura argumental como un punto axial para la organización oracional y la reconstrucción de los protagonistas textuales. La manipulación consciente de las estructuras sintácticas en relación con las interpretaciones semánticas asociadas potencia la competencia lingüística del alumnado propiciando reflexiones que ponen de manifiesto las estrategias de poder ocultas en los mecanismos discursivos. Las intervenciones pragmáticas necesariamente requieren del acompañamiento y el detenimiento en el nivel oracional. El trabajo conjunto de los niveles morfosintáctico y semántico de la frase y la oración resultan un camino seguro hacia la posibilidad de analizar estructuras pragmáticas como las implicaturas y los presupuestos.

El contenido "verbo", presente en la selección curricular de los docentes, resulta opacado por la centralidad de los contenidos textuales. Así, la reflexión gramatical sobre el verbo es posterior al análisis textual y este análisis es lo que lo relega al lugar de normativa, al lugar de reglas a cumplir o a respetar en la construcción de determinados textos. La atención puesta exclusivamente en el texto no aportó mejoras en el nivel de producción y comprensión textual, uno de los grandes objetivos del área de lengua.

Según se mostró en el escrito, cuando se incluye el contenido gramatical "verbo" en las propuestas de clase, su lugar es restringido, limitado a su reconocimiento de función núcleo del predicado en el análisis oracional, muchas veces sin una reflexión gramatical previa.

Para llegar a reflexionar sobre la construcción de los textos es necesario objetivar el lenguaje para crear un metalenguaje compartido entre el alumnado y el docente. Es 
preciso contar con un conocimiento compartido para que la enseñanza no sea contenidista, para que el conocimiento no se vuelva un "conocimiento ritual” (Perkins, I992: 36) que es evidencia de que el conocimiento es “frágil”. En la mayoría de los casos el análisis de oraciones tiene en las aulas un carácter repetitivo. Este carácter repetitivo sin explicación es lo que se convierte para el alumnado en conocimiento ritual, un conocimiento que sólo sirve "para cumplir con las tareas escolares." (Perkins, I992: 37)

En este sentido, consideramos necesario revisar la solidez de la formación disciplinar de los docentes a fin de garantizar la toma de decisiones didácticas y epistemológicas, en relación con el objeto de enseñanza, que se basen en una argumentación razonada y a posteriori de consideraciones respecto de las potencialidades de las diferentes miradas sobre el objeto. Lo dicho hasta aquí no implica descuidar la formación pedagógica y la didáctica sino, por el contrario, cuidar que en la conjugación entre estos saberes necesarios para el ejercicio de la docencia, no se banalicen o distorsionen los contenidos. 


\section{Referencias bibliográficas}

- AAVV. (I994). Ley Federal de Educación, N²4.I25, Ministerio de Cultura y Educación de la Nación. Argentina.

- AAVV. (2006). Ley de Educación Nacional, $N^{\circ}$ 26.206. Ministerio de Educación, Ciencia y Tecnología. Argentina.

-Alisedo, G.; Melgar, S. y Chiocci, C. (i994). Didáctica de las ciencias del lenguaje. Aportes y reflexiones. Buenos Aires: Paidós Educador.

- Bombini, G. (2OOI). Avatares en la configuración de un campo: la didáctica de la lengua y la literatura. Lulú Coquette. Revista de didáctica de la lengua y la literatura, (Año I, № I). 24-33. Buenos Aires.

- Carrió, C. (2005). El debate en torno a la enseñanza de la lengua: el lugar de la gramática. Actas del IV Congreso de las Lenguas del MERCOSUR. Lenguaje y Multidisciplinariedad. I5O-I57. Chaco: Universidad Nacional del Nordeste.

- Carrió, C. (2008). Entre Nombres y Sobrenombres. Anais del Encontro Internacional de Pesquisadores de Políticas Educativas. I83-I87. Porto Alegre: Editorial Grupo Montevideo (AUGM) - Facultade Rio Grande do Sul.

- Carrió, C. y Guglielmelli, J. (2OII). Repensando las relaciones entre semántica y pragmática. Notas para discutir. En A. Gerbaudo (Dir.). La lengua y la literatura en la escuela secundaria (pp. 203-2I3). Santa Fe-Rosario: UNL-Homo Sapiens.

- Castellà, J. (I994). ¿Qué gramática para la escuela? Sobre árboles, gramáticas y otras formas de andarse por las ramas. En TEXTOS de didáctica de la lengua y la literatura (I5-24). Barcelona: Graó Educación.

- Celman, S.; Galarraga, G.; Gerard, A.; Grinóvero, N.; Martínez, M.; Olmedo, V. y Rafaghelli, M. (2OI3). El complejo caso de la evaluación educativa. Sentidos y prácticas construidas. En S. Celman; G. Galarraga; A. Gerard; N. Grinóvero; M. Martínez; V. Olmedo y M. Rafaghelli (Comps.). Evaluaciones. Experiencias entre la universidad pública y los Institutos de Formación Docente (pp. II-36). Paraná: EDUNER.

- Ciminari, L. y Kocak, C. (2002). Aportes para una didáctica del léxico. Presentado en el I Congreso Internacional de Educación. Lenguaje y Sociedad. Tensiones educativas en América Latina. 
- Defagó, C. (2002). No siempre lo simple es lo más sencillo. Una reflexión sobre la gramática en la escuela. En G. Herrera de Bett (comp.). Didácticas de la lengua y la literatura. Teorías, debates y propuestas. Córdoba: Universidad Nacional de Córdoba.

- Defagó, C. (2OII). El lenguaje como conocimiento: aportes para la didáctica de la lengua. En A. Gerbaudo (Dir.). La lengua y la literatura en la escuela secundaria (pp. I87-202). Santa Fe-Rosario: UNL-Homo Sapiens.

- Defagó, C. y Guglielmelli, J. (2005). La reflexión gramatical. De lo implícito a lo explícito en la enseñanza de la lengua. Presentado en las II Jornadas Internacionales de Educación Lingüistica. Concordia: UNER.

- Demonte, V. (2005). La gramática ubicua. O cómo se miran los textos a través de la gramática. Obtenido el 22 de octubre de 2015 desde https://www.uam.es/personal_pdi/filoyletras/vdemonte/ubicua.pdf

- Desinano, N. (1997). Didáctica de la lengua para elıer. Ciclo EGB. Rosario: Homo Sapiens.

- Di Tullio, Á. (1998). Manual de gramática del español. Argentina: Edicial.

- Fenstermacher, G. (I986). Tres aspectos de la filosofía de la investigación sobre la enseñanza. En M. Wittrock (Ed.). La investigación de la enseñanza, I. Enfoques, teorías y métodos (pp. I49-I79). Buenos Aires: Paidós.

- Giamatteo, M. y Albano, H. (2009). ¿Cómo se clasifican las palabras? Buenos Aires: Biblos.

- Gerbaudo, A. (2006). Ni dioses ni bichos. Profesores de literatura, currículum y mercado. Santa Fe: UNL.

- Gerbaudo, A. (2OII). El docente como autor del currículum: una reinstalación política y teórica necesaria. En A. Gerbaudo (Dir.). La lengua y la literatura en la escuela secundaria (pp. I7-27). Santa Fe-Rosario: UNL-Homo Sapiens.

- Llambí de Adra, M. E. (2005). Aportes para redefinir el valor del léxico en la enseñanza de la lengua. Obtenido el 22 de octubre de 2015 desde http://www.educ.ar/sitios/educar/ recursos/ver?id=9I3I8 (22/OI/2OI5)

- Litwin, E. (I998). La evaluación: campo de controversias y paradojas o un nuevo lugar para la buena enseñanza. En A. Camilloni; S. Celman; E. Litwin y M. Palau de Maté. La evaluación de los aprendizajes en el debate didáctico contemporáneo (pp. II-33). Buenos Aires: Paidós. 
- Lorenzotti, M. (2OI6). La enseñanza del "verbo" en el aula de lengua: diálogo con las propuestas curriculares Nacionales y Jurisdiccionales. Santa Fe. (mimeo)

- Otañi, I. y Gaspar, M. del P. (200I). Sobre la Gramática. En M. Alvarado (Coord.). Entre líneas. Teorías y enfoques en la enseñanza de la escritura, la gramática y la literatura (pp. 75-III). Buenos Aires: FLACSO Manantial.

- Perkins, D. (1992). La escuela inteligente. Del adiestramiento de la memoria a la educación de la mente. Barcelona: Gedisa. I998. 